\title{
Outage-optimal opportunistic scheduling with analog network coding in multiuser two-way relay networks
}

\author{
Prabhat K Upadhyay ${ }^{* *}$ and Shankar Prakriya ${ }^{2}$
}

\begin{abstract}
This paper investigates the performance of an outage-optimal opportunistic scheduling scheme for a multiuser two-way relay network, wherein an analog network coding-based relay serves multiple pairs of users. Under a Rayleigh flat-fading environment, we derive an exact expression for cumulative distribution function (CDF) of the minimum of the two end-to-end instantaneous signal-to-noise ratios (SNRs) and utilize this to obtain an exact expression for the outage probability of such a greedy scheduling scheme. We then develop a modified scheduler that ensures fairness among user pairs of the considered system. By using a high SNR approximation of derived CDF, we present a simple closed-form expression for outage probability of the overall system and establish that a multiuser diversity of order equal to the number of user pairs is harnessed by the scheme. We also present an efficient power allocation strategy between sources and relay, subject to a total power constraint, that minimizes the outage probability of the overall system. Further, by deriving both upper and lower bound expressions for the average sum-rate of the proposed scheme, we demonstrate that an average sum-rate gain can also be achieved by increasing the number of user pairs in the system. Numerical and simulation results are presented to validate the performance of the proposed scheme.
\end{abstract}

Keywords: Analog network coding, Multiuser scheduling, Outage probability, Rayleigh fading, Two-way (bidirectional) relaying

\section{Introduction}

Cooperative relaying techniques have recently gained great research interest because of their potential in enhancing the throughput or reliability of wireless networks. Several schemes have been extensively studied in literature to achieve cooperative diversity utilizing the one-way relaying protocol [1]. However, the half-duplex constraint at the relays incurs a spectral efficiency loss in such schemes. Recent research has shown that such a loss can be effectively mitigated by exploiting the idea of network coding [2] in bidirectional communication scenarios [3-7]. Bidirectional cooperative relaying strategies facilitate information exchange between two users in either four, three, or two time phases via a half-duplex relay. The four-phase protocol follows the conventional

\footnotetext{
* Correspondence: pkupadhyay@ee.iitd.ac.in

'Department of Electrical Engineering, Indian Institute of Technology Delhi, New Delhi, 110016, India

Full list of author information is available at the end of the article
}

approach by requiring two separate time phases for data flow in each direction and hence is spectrally inefficient. However, the bidirectional communication has been shown to be accomplished in even three phases in [3-7]. In the three-phase protocol (called physical layer network coding (PNC) [6] or time division broadcast (TDBC) [7]), the two users transmit successively in first and second phases, the relay then decodes both the data, applies network coding, and forwards the combined data to both users in the third phase. After canceling the self-interferences (as they are known by the respective users), the intended message can be received at each of the user terminals. Recently, a two-way relaying protocol $[8,9]$ has emerged as a promising technique to mitigate the spectral efficiency loss of conventional half-duplex relaying systems. In this scheme, the two users communicate bidirectionally (in the absence of a reliable direct link) in just two time phases, namely the multiple access channel (MAC) phase and the broadcast 
Table 1 Selected values of channel variances over the two hops for i.ni.d. user pairs

\begin{tabular}{cc}
\hline$\left.\sigma_{a, k}^{2}\right\}_{k=1}^{K}$ & $\left\{\sigma_{b, k}^{2}\right\}_{k=1}^{K}$ \\
\hline 0.2 & 0.5 \\
0.8 & 0.3 \\
0.4 & 0.1 \\
0.9 & 0.7 \\
0.4 & 0.8 \\
0.2 & 0.3 \\
0.9 & 0.5 \\
0.1 & 0.7 \\
0.7 & 0.2 \\
0.2 & 0.9 \\
0.3 & 0.4 \\
0.6 & 0.5 \\
0.8 & 0.2 \\
0.5 & 0.3 \\
0.2 & 0.6 \\
0.4 & 0.7 \\
\hline
\end{tabular}

channel (BC) phase. In the first phase (MAC), both users transmit their data simultaneously to the relay, and the relay broadcasts the processed signal to both users in the second phase $(\mathrm{BC})$. When an amplify-andforward (AF) processing is applied on the superimposed signal received during the MAC phase at the relay, such a scheme is usually termed as analog network coding (ANC) [10-13].

The two-phase two-way relaying protocol has also been generalized to a multiuser scenario in which multiple pairs of users communicate bidirection-ally via one or more relays $[8,14-18]$. The authors in $[8,14]$ considered several relays or antennas that orthogonalize multiple pairs by a distributed zero-forcing technique. A spread spectrum based interference management scheme wherein each pair shares a common spreading signature, and the relay uses a jointly demodulate-andXOR forward strategy is proposed in [15]. The information theoretic capacity for such a scheme is studied in [16] and [17] by considering a deterministic channel model and a Gaussian two-pair two-way full-duplex relay network, respectively. To combat interference at each user of such a system, the authors in [18] proposed different beamforming schemes with amplify-and-forward (AF) and quantize-and-forward (QF) strategies at the relay. However, to the best of our knowledge, a performance analysis exploiting multiuser diversity for this system has not been reported so far. Although the twophase two-way relaying protocol is spectrally efficient, it incurs a penalty in diversity as compared to conventional one-way relaying [1] due to the absence of the direct path. In traditional wireless communications under a multiuser downlink scenario, it has been shown in many publications $[19,20]$ that opportunistic scheduling of users can provide diversity gains. Therefore, the use of scheduling to harness multiuser diversity is well motivated in the two-way relaying context. Note that such opportunistic access avoids the difficult synchronization issues associated with simultaneous transmission of multiple user pairs, as well as the requirement of large number of antennas at the relay. However, scheduling strategies for such two-way systems are different from the commonly studied downlink scenarios. For this reason, development of good scheduling strategies in the two-way context is of considerable interest.

With the above motivation, we propose in this paper an opportunistic scheduling scheme for a multi-pair ANC-based two-way relay system and evaluate its performance over Rayleigh fading channels. We first consider a greedy scheduler based on minimizing the overall outage probability of the system. Considering channel state information (CSI)- and noise statisticsassisted gain at the relay, we derive an exact expression for the CDF of the minimum of SNRs of the two endto-end transmissions, which is applicable for the whole SNR region. This facilitates an exact outage performance analysis for the considered greedy scheduling scheme. We then propose a scheduler that ensures fairness among user pairs of the system. By using a high SNR approximation of derived CDF, we obtain a simple closed-form expression for the outage probability of the overall system. Further, we provide an efficient power allocation scheme based on the derived expression that minimizes the system outage probability. In addition, we derive expressions for upper and lower bounds on the average sum-rate of the considered system. Numerical and simulation results illustrate the effectiveness of our analytically derived results and show that the considered scheme achieves performance gain by attaining an order of multiuser diversity equal to the number of user pairs.

The rest of the paper is organized as follows: Section 2 describes the system model. The opportunistic scheduling criteria are formulated in Section 3. The overall system is analyzed in terms of access probability, outage probability, and average sum-rate under Rayleigh fading in Section 4. Section 5 presents numerical and simulation results, and finally, Section 6 concludes the paper.

\section{Notation}

We use $z \sim \mathcal{C N}\left(\mu, \sigma^{2}\right)$ to denote a complex circular Gaussian random variable $z$ with mean $\mu$ and variance $\sigma^{2}$. The operators $\mathbb{E}[\cdot], \operatorname{Pr}[\cdot]$, and $|\cdot|$ represent expectation, probability, and absolute value, respectively. (.) denotes the binomial coefficient, $\ln (\cdot)$ denotes the natural $\log$ arithm, and $\log (\cdot)$ refers to $\log _{2}(\cdot)$. 


\section{System descriptions}

We consider a multiuser two-way relay network consisting of $2 K+1$ single-antenna nodes ( $K$ pairs of users and one relay), as depicted in Figure 1. Nodes $T_{a, k}$ and $T_{b, k}$ denote members of $k$ th pair, $k \in\{1,2, \ldots, K\}$, that want to communicate bidirectionally via a single relay $R$. All nodes operate in a half-duplex fashion. The communication takes place slot-wise where one time-slot represents the end-to-end transmission duration. In what follows, we consider one time-slot to be comprised of two phases of equal duration, viz., the MAC phase and the $\mathrm{BC}$ phase. We assume that the channels for all links are subject to independent, but not necessarily identical frequency-flat Rayleigh fading. Let $h_{l, k}(t)$ denote the channel fading coefficient between node $T_{l, k}$ and relay $R$ during the th time-slot, where $l \in\{a, b\}$. We adopt the quasi-static fading-channel model such that $h_{l, k}(t)$ remains constant during a time-slot but independently changes in different time-slots. Since the scheduling policy is determined in each slot, we drop the time index $t$ for notational simplicity. Therefore, $h_{l, k}$ can be modeled as $\mathcal{C N}\left(0, \sigma_{l, k}^{2}\right)$ where $\sigma_{l, k}^{2}$ is the average fading power of the link between $T_{l, k}$ and $R$. We further assume that the relay $R$ has perfect global channel state information (CSI) of the network. To facilitate CSI estimation at the users, the relay periodically broadcasts a common pilot signal to all users. Then, each user feeds back that CSI to the relay and assuming channel reciprocity, a scheduling strategy for opportunistic transmission by user pairs can be employed. The user pairs are informed about the scheduling decision through a certain control signal by the relay. The specific scheduling policy will be elaborated upon in Section 3.

Let us consider that the $k$ th user pair is scheduled for transmission and has access to relay channel resources for a given time-slot. We focus on the two-step ANC protocol whereby the information exchange for the $k$ th pair takes place in two phases of equal time duration. In the first phase (MAC), both users of $k$ th pair simultaneously transmit their data to the relay with equal power $P$. Note that equal transmit power assumption at

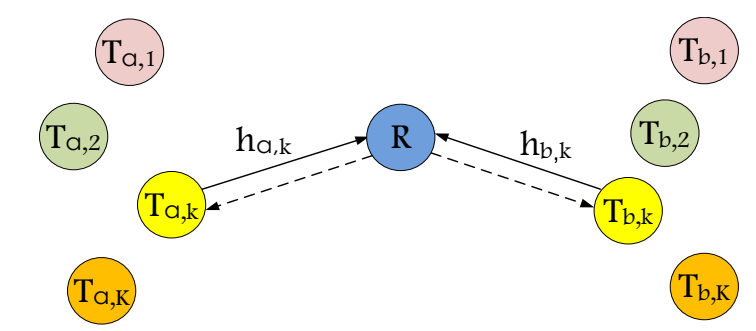

Figure $1 \mathrm{~K}$-pair two-way relaying system. The solid and broken line arrows indicate data transmissions in orthogonal phases (MAC and $\mathrm{BC}$ respectively). the users does not loose generality in diversity performance analysis, as its effect can be included in the average SNR of each channel. With this, the received signal at the relay is given by

$$
y_{r, k}=\sqrt{P} h_{a, k} x_{a, k}+\sqrt{P} h_{b, k} x_{b, k}+n_{r, k}
$$

where $x_{a, k}$ and $x_{b, k}$ are the transmit symbols having unit energy from the sources $T_{a, k}$ and $T_{b, k}$, respectively, and $n_{r, k}$ is an additive white Gaussian noise (AWGN) at the relay. The AWGN at all nodes is assumed as independent and identically distributed (i.i.d.) $\mathcal{C N}\left(0, N_{0}\right)$ with the noise variance per dimension is $N_{0} / 2$. Therefore, we define $P / N_{0}$ as simply the SNR.

During the second phase $(\mathrm{BC})$, the signals received at the destinations $T_{a, k}$ and $T_{b, k}$ via the AF relay can be expressed, respectively, as

$$
y_{a, k}=\beta_{k} \sqrt{P} h_{a, k} h_{a, k} x_{a, k}+\beta_{k} \sqrt{p} h_{a, k} h_{b, k} x_{b, k}+\beta_{k} h_{a, k} n_{r, k}+n_{a, k}
$$

and

$$
y_{b, k}=\beta_{k} \sqrt{P} h_{a, k} h_{b, k} x_{a, k}+\beta_{k} \sqrt{p} h_{b, k} h_{b, k} x_{b, k}+\beta_{k} h_{b, k} n_{r, k}+n_{b, k}(3)
$$

where $n_{a, k}$ and $n_{b, k}$ denote AWGN at the nodes $T_{a, k}$ and $T_{b, k}$, respectively, and $\beta_{k}$ represents the power-constrained amplifying gain at the relay given by

$$
\beta_{k}=\sqrt{\frac{P_{r}}{P\left|h_{a, k}\right|^{2}+P\left|h_{b, k}\right|^{2}+N_{0}}},
$$

where $P_{r}$ is the transmit power at the relay. The intrapair interferences in (2) and (3) can be canceled out as they are known at the respective terminals, and hence, the received signals can be re-expressed as

$$
\tilde{y}_{a, k}=\beta_{k} \sqrt{P} h_{a, k} h_{b, k} x_{b, k}+\beta_{k} h_{a, k} n_{r, k}+n_{a, k}
$$

and

$$
\tilde{y}_{b, k}=\beta_{k} \sqrt{P} h_{a, k} h_{b, k} x_{a, k}+\beta_{k} h_{b, k} n_{r, k}+n_{b, k} .
$$

The resultant end-to-end instantaneous SNRs at nodes $T_{a, k}$ and $T_{b, k}$ are given, respectively, by

$$
\gamma_{a, k}=\frac{P_{r} P}{N_{0}}\left[\frac{\left|h_{a, k}\right|^{2}\left|h_{b, k}\right|^{2}}{\left(P_{r}+P\right)\left|h_{a, k}\right|^{2}+P\left|h_{b, k}\right|^{2}+N_{0}}\right]
$$

and

$$
\gamma_{b, k}=\frac{P_{r} P}{N_{0}}\left[\frac{\left|h_{a, k}\right|^{2}\left|h_{b, k}\right|^{2}}{\left(P_{r}+P\right)\left|h_{b, k}\right|^{2}+P\left|h_{a, k}\right|^{2}+N_{0}}\right] .
$$

The corresponding one-sided data-rates are thus given as $\quad \mathcal{R}_{a, k}=\frac{1}{2} \log \left(1+\gamma_{a, k}\right) \quad$ and $\quad \mathcal{R}_{b, k}=\frac{1}{2} \log \left(1+\gamma_{b, k}\right)$. Finally, the sum-rate of $k$ th pair opportunistic transmission is given by 


$$
\begin{aligned}
\mathcal{R}_{k}^{\text {sum }} & =\mathcal{R}_{a, k}+\mathcal{R}_{b, k} \\
& =\frac{1}{2} \log \left[\left(1+\gamma_{a, k}\right)\left(1+\gamma_{b, k}\right)\right] .
\end{aligned}
$$

Note that the pre-log factor $\frac{1}{2}$ accounts for the fact that intra-pair information exchange takes place in two time phases.

\section{Opportunistic scheduling strategy}

In this section, we explain a multi-pair scheduling strategy and suggest a selection criteria for the best user pair for the system discussed previously. With perfect global CSI knowledge, the relay determines to service a target user pair in every time-slot. The key issue is how to determine the appropriate metric for channel-aware scheduling among user pairs. We first consider a greedy scheduling policy in which the best user pair $k^{*}$ is chosen among multiple pairs in each time-slot based on the following criterion:

$$
k^{*}=\arg \max _{k \in \mathcal{K}}\left\{\theta_{k}\right\},
$$

where $\theta_{k}=\min \left(\gamma_{a, k}, \gamma_{b, k}\right)$ and $\mathcal{K}=\{1,2, \ldots, K\}$ is the set of user pairs. However, by using the expressions of $\gamma_{a, k}$ and $\gamma_{b, k}$ given in (7) and (8), respectively, at a high SNR, one can recognize that the scheduling policy of (10) is equivalent to

$$
k^{*}=\arg \max _{k \in \mathcal{K}}\left\{\phi_{k}\right\},
$$

where $\varphi_{k}=\min \left(\left|h_{a, k}\right|^{2},\left|h_{b, k}\right|^{2}\right)$.

The aforementioned greedy scheduling strategy thus selects the user pair for each time-slot with the largest value of the smaller end-to-end instantaneous SNRs. However, users' channels usually have different statistics due to different locations, and a user pair whose terminals are situated far away from the relay is unlikely to be ever selected by the scheduler. Hence, such a greedy scheduling scheme leads to an unfair resource allocation among user pairs, particularly for the case when the pairs are independent and non-identically distributed (i. ni.d.). To address this issue of fairness, the scheduling policy we now propose to use selects the best user pair $k^{*}$ in each time-slot based on the following criterion:

$$
k^{*}=\arg \max _{k \in \mathcal{K}}\left\{\frac{\theta_{k}}{\bar{\theta}_{k}}\right\},
$$

where $\bar{\theta}_{k}$ is the average value of $\theta_{k}$ for $k$ th user pair in the given time-slot. Normalization by $\bar{\theta}_{k}$ in (12) is used in order to maintain long-term fairness among user pairs. To facilitate this, the relay keeps updating $\bar{\theta}_{k}$ in each time-slot. It is not difficult to realize that the pairs having poor channel quality may not have to wait longer to gain access to the relay channel. Considering now that user pairs are independent and identically distributed (i.i.d.), that is, the average values of $\theta_{k}$ are identical such that $\bar{\theta}_{k}=\bar{\theta}$ for all $k$, the scheduler policy in (12) is reduced to that stated in (10).

Next, we investigate the performance of the system discussed previously based on multi-pair scheduling policy stated in (10)-(12) in the presence of Rayleigh fading.

\section{Performance analysis}

First of all, we derive an exact expression for the cumulative distribution function (CDF) of $\theta_{k}$. Then, by approximating the derived CDF in simple closed-form at high SNR, we obtain the expressions for the probability density function (PDF) and the CDF of best user pair as defined in (12) for the fair scheduling scheme. Finally, we analyze the overall system performance in terms of access probability, outage probability, and average sumrate.

Under Rayleigh fading, $\left|h_{a, k}\right|^{2}$ and $\left|h_{b, k}\right|^{2}$ for any $k \in \mathcal{K}$ are independent but not necessarily identically distributed exponential random variables with parameters $1 / \sigma_{a, k}^{2}$ and $1 / \sigma_{b, k}^{2}$, respectively. An exact expression for the CDF of $\theta_{k}$ is provided in the following theorem.

Theorem 1 The CDF $F_{\theta_{k}}(\theta)$ of $\theta_{k}$ for any $k \in \mathcal{K}$ is given by

$$
\begin{aligned}
F_{\theta_{k}}(\theta) & =\operatorname{Pr}\left[\min \left(\gamma_{a, k}, \gamma_{b, k}\right)<\theta\right] \\
& =P_{1, k}+P_{2, k},
\end{aligned}
$$

where $P_{1, k}$ and $P_{2, k}$ are given, respectively, by

$$
\begin{aligned}
P_{1, k}=1- & \frac{\eta \lambda_{y_{1, k}}}{\lambda_{x_{1, k}}+\eta \lambda_{\gamma_{1, k}}}\left[1-e^{-\frac{\delta}{\eta}\left(\lambda_{x_{1, k}}+\eta \lambda_{\gamma_{1, k}}\right)}\right] \\
& -\lambda_{y_{1, k}} e^{-\gamma\left(\lambda_{x_{1, k}}+\lambda_{1_{1, k}}\right)} \sum_{n=0}^{\infty} \frac{(-1)^{n}}{n !} \frac{\left[\lambda_{x_{1, k}} \gamma(\gamma+1)\right]^{n}}{(\delta-\gamma)^{n-1}} E_{n}\left[\lambda_{\gamma_{1, k}}(\delta-\gamma)\right]
\end{aligned}
$$

and

$$
\begin{aligned}
P_{2, k}=1- & \frac{\eta \lambda_{x_{2, k}}}{\lambda_{y_{2, k}}+\eta \lambda_{x_{2, k}}}\left[1-e^{-\frac{\delta}{\eta}\left(\lambda_{\gamma_{2, k}}+\eta \lambda_{x_{2, k}}\right)}\right] \\
& -\lambda_{x_{2, k}} \mathrm{e}^{-\gamma\left(\lambda_{\gamma_{2, k}}+\lambda_{x_{2, k}}\right)} \sum_{n=0}^{\infty} \frac{(-1)^{n}}{n !} \frac{\left[\lambda_{\gamma_{2, k}} \gamma(\gamma+1)\right]^{n}}{(\delta-\gamma)^{n-1}} E_{n}\left[\lambda_{x_{2, k}}(\delta-\gamma)\right]
\end{aligned}
$$

with

$\lambda_{x_{1, k}}=N_{0} /\left(P \sigma_{a, k}^{2}\right), \lambda_{\gamma_{1, k}}=N_{0} /\left(\left(P+P_{r}\right) \sigma_{b, k}^{2}\right), \lambda_{x_{2, k}}=N_{0} /\left(\left(P+P_{r}\right) \sigma_{a, k}^{2}\right), \lambda_{y_{2, k}}=N_{0} /\left(P \sigma_{b, k}^{2}\right),=\theta\left(P+P_{r}\right) / P_{r}, \eta=\left(P+P_{r}\right) / P$, and $\delta=\frac{1}{2}\left[\gamma(\eta+1)+\sqrt{\gamma^{2}(\eta+1)^{2}+4 \gamma \eta}\right] . E_{n}[z]$ denotes the exponential integral of order $n$ defined in [21, eq. 5.1.4] as $E_{n}[z]=\int_{1}^{\infty} t^{-n} e^{-z t} \mathrm{~d} t$.

See "Appendix I" for the proof of Theorem 1. It is worth noting that the expressions in (14) and (15) involve only exponentials and exponential integral functions. These can be numerically evaluated with sufficient accuracy using symbolic software packages such as 
MATHEMATICA and MATLAB. Further, the single infinite series expansion in (14) or (15) can be represented as $\sum_{n=0}^{\infty} \Lambda_{n}$ where $\Lambda_{n}=\frac{(-1)^{n}}{n !} \frac{[u \gamma(\gamma+1)]^{n}}{[\delta-\gamma]^{n-1}} E_{n}[\nu(\delta-\gamma)] \times v e^{-\gamma(u+v)}$ denoting the $n$-th term with $u, v \in\left\{\lambda_{x_{1, k}}, \lambda_{x_{2, k}}, \lambda_{y_{1, k}}, \lambda_{y_{2, k}}\right\}$ and $u \neq v$, for and $k$. Since $E_{n}[z]$ decreases monotonically with $n$, it can be shown that $\lim _{n \rightarrow \infty}\left|\frac{\Lambda_{n+1}}{\Lambda_{n}}\right|=\lim _{n \rightarrow \infty} \frac{u \gamma(\gamma+1)}{(\delta-\eta)(n+1)} \frac{E_{n+1}[v(\delta-\gamma)]}{E_{n}[v(\delta-\gamma)]}<1$, satisfying the convergence criteria as per the ratio test [22].

Although the expression given by (13) is exact and valid for all values of SNR, it is difficult to facilitate in particular the analysis for the case of fairness in scheduling scheme. We hence focus on deriving a simple closed-form expression of $F_{\theta_{k}}(\theta)$ at high SNR $\left(P \gg N_{0}\right)$ in the following Lemma.

Lemma 1 The $C D F F_{\theta_{k}}(\theta)$ of $\theta_{k}$ can be approximated at high SNR as

$$
F_{\theta_{k}}(\theta) \approx 1-e^{-\frac{\eta N_{0} \theta}{P_{r}}\left(\frac{1}{\sigma_{a, k}^{2}}+\frac{1}{\sigma_{b, k}^{2}}\right)} .
$$

See "Appendix II" for the proof of Lemma 1.

Note that such an approximate expression yields very tight results in the whole SNR region and therefore can be used to make analysis feasible for the case of fairness in scheduling. We make here an interesting remark that $\theta_{k}$ in (16) follows an exponential distribution with its mean value $\bar{\theta}_{k}=\frac{P_{r} \sigma_{a, k}^{2} \sigma_{b, k}^{2}}{\eta N_{0}\left(\sigma_{a, k}^{2}+\sigma_{b, k}^{2}\right)}$. Moreover, $\varphi_{k}$ in (11) is also exponentially distributed with CDF given by

$$
F_{\phi_{k}}(\phi)=1-e^{-\phi\left(\frac{1}{\sigma_{a, k}^{2}}+\frac{1}{\sigma_{b, k}^{2}}\right)},
$$

where the mean value of $\varphi_{k}$ is given by $\bar{\phi}_{k}=\frac{\sigma_{a, k}^{2} \sigma_{b ; k}^{2}}{\sigma_{a, k}^{2}+\sigma_{b, k}^{2}}$.

Now applying a similar method as in [20], developed for downlink multiuser systems, we can express the PDF and the CDF of the best user pair (with $\theta_{k^{\prime \prime}}$ ) for the considered fair scheduling system, respectively, as

$$
f_{\theta_{k *}}(\theta)=\sum_{k=1}^{K} \frac{1}{\bar{\theta}_{k}} f_{k}\left(\frac{\theta}{\bar{\theta}_{k}}\right) \prod_{\substack{j=1 \\ j \neq k}}^{K} F_{j}\left(\frac{\theta}{\bar{\theta}_{k}}\right)
$$

and

$$
F_{\theta_{k^{*}}}(\theta)=\sum_{k=1}^{K} \int_{0}^{\frac{\theta}{\bar{\theta}_{k}}} f_{k}(x) \prod_{\substack{j=1 \\ j \neq k}}^{K} F_{j}(x) \mathrm{d} x,
$$

where $f_{k}(\cdot)$ and $F_{k}(\cdot)$ are the PDF and the CDF of the normalized variable $\theta_{k} / \bar{\theta}_{k}$ for the $k$ th pair, respectively. Using (16), we can express (18) and (19), respectively, as

$$
f_{\theta_{k^{*}}}(\theta) \simeq \sum_{k=1}^{K} \frac{1}{\bar{\theta}_{k}} \sum_{n=0}^{K-1}\left(\begin{array}{c}
K-1 \\
n
\end{array}\right)(-1)^{n} e^{-(n+1) \theta / \bar{\theta}_{k}}
$$

and

$$
F_{\theta_{k^{*}}} \simeq \frac{1}{K} \sum_{k=1}^{K}\left(1-\mathrm{e}^{-\theta / \bar{\theta}_{k}}\right)^{K},
$$

where the $\simeq$ sign denotes the equality in the region of high SNR.

\subsection{Access probability}

The access probability can be defined as the probability that user pair $k \in \mathcal{K}$ accesses the relay channel in the long run. It can be expressed by

[20]

$$
\begin{aligned}
P_{k}^{\mathrm{acc}} & =\operatorname{Pr}\left[\frac{\theta_{k}}{\bar{\theta}_{k}} \geq \frac{\theta_{j}}{\bar{\theta}_{j}} \quad \forall \quad j \neq k\right] \\
& =\int_{0}^{\infty} \frac{1}{\bar{\theta}_{k}} f_{k}\left(\frac{\theta}{\bar{\theta}_{k}}\right) \prod_{\substack{j=1 \\
j \neq k}}^{K} F_{j}\left(\frac{\theta}{\bar{\theta}_{k}}\right) d \theta .
\end{aligned}
$$

We can evaluate (22) by using (16) as

$$
\begin{aligned}
P_{k}^{\mathrm{acc}} & \simeq \sum_{m=0}^{K-1}\left(\begin{array}{c}
K-1 \\
m
\end{array}\right) \frac{(-1)^{m}}{m+1} \\
& =\frac{1}{K} \sum_{m=0}^{K-1}\left(\begin{array}{c}
K \\
m+1
\end{array}\right)(-1)^{m}=\frac{1}{K} .
\end{aligned}
$$

Thus, as expected, the scheduling policy in (12) is fair in the sense that each pair $k$ can have equal access probability of $1 / K$.

\subsection{Outage probability}

For each user pair, an end-to-end transmission is in outage when either user of the pair is in outage, that is, when either $\mathcal{R}_{a, k}$ or $\mathcal{R}_{b, k}$ is smaller than the target rate $\mathcal{R}$. Hence, the outage probability for the best pair $k^{*}$ is given by

$$
\begin{aligned}
P_{k^{*}}^{\text {out }} & =\operatorname{Pr}\left[\mathcal{R}_{a, k^{*}}<\mathcal{R} \text { or } \mathcal{R}_{b, k^{*}}<\mathcal{R}\right] \\
& =\operatorname{Pr}\left[\min \left(\gamma_{a, k^{*}}, \gamma_{b, k^{*}}\right)<\gamma_{\text {th }}\right],
\end{aligned}
$$

where $\gamma_{\text {th }}=2^{2 \mathcal{R}}-1$ is a threshold required for successful decoding at the receiver(s). As such, it is obvious that the considered greedy scheduling scheme minimizes the system outage probability. 
Using the definition of best user pair for greedy scheduling scheme in (10) and applying the theory of order statistics [23] with $K$ i.ni.d. random variables, the outage probability in (24) is given as

$$
P_{k^{*} \text {, greedy }}^{\text {out }}=\prod_{k=1}^{K} F_{\theta_{k}}\left(\gamma_{\text {th }}\right) \text {, }
$$

which can be calculated exactly by using the CDF of $\theta_{k}$ as given in (13).

For the fair scheduling scheme stated in (12), we can express the outage probability in (24) by using (21) as

$$
\begin{aligned}
& P_{k^{*}}^{\text {out }}=F_{\theta_{k^{*}}}\left(\gamma_{\text {th }}\right) \\
& \simeq \frac{1}{K} \sum_{k=1}^{K}\left[1-e^{-\frac{\eta N_{0} \gamma_{\mathrm{th}}}{P_{r}}\left(\frac{1}{\sigma_{a, k}^{2}}+\frac{1}{\sigma_{b, k}^{2}}\right)}\right]^{K} .
\end{aligned}
$$

We now address an efficient power allocation problem to the relay subject to a total power constraint. Specifically for the total end-to-end transmission power $P_{t}=2 P+P_{r}$, we consider $P_{r}=\alpha P_{t}$ and $P=\left(\frac{1-\alpha}{2}\right) P_{t}$ where $\alpha \in$

denotes the fraction of total power $P_{t}$ allocated to the relay. Henceforth, we define $\mathrm{e}=P_{t} / N_{0}$ as the total SNR. With such power distribution, we can rewrite (26) as

$$
P_{k^{*}}^{\text {out }} \simeq \frac{1}{K} \sum_{k=1}^{K}\left[1-\mathrm{e}^{-\frac{(1+\alpha) \gamma_{\text {th }}}{\alpha(1-\alpha) \varrho}\left(\frac{1}{\sigma_{a, k}^{2}}+\frac{1}{\sigma_{b, k}^{2}}\right)}\right]^{K} .
$$

Now, we can show that the expression of outage probability in (27) is minimized when $\alpha=\sqrt{2}-1 \approx 0.414$. It is important to emphasize that this power allocation is independent of $\sigma_{a, k}^{2}$ and $\sigma_{b, k}^{2}$ for all $k$.

To investigate the asymptotic outage behavior, we can re-express (27) at high total SNR $(\varrho \rightarrow \infty)$ as

$$
P_{k^{*}}^{\text {out }} \simeq \frac{1}{K}\left[\frac{(1+\alpha) \gamma_{\mathrm{th}}}{\alpha(1-\alpha) \varrho}\right]^{K} \sum_{k=1}^{K}\left(\frac{1}{\sigma_{a, k}^{2}}+\frac{1}{\sigma_{b, k}^{2}}\right)^{k},
$$

which follows from the approximation $e^{-z} \underset{z \rightarrow 0}{\approx} 1-z$. By using the definition of diversity order as $d=-\lim _{\varrho \rightarrow \infty} \frac{\log \left[P_{k^{*}}^{\text {out }}(\varrho)\right]}{\log \varrho}$, we can verify that the proposed scheduling scheme can achieve a multiuser diversity of order $K$.

\subsection{Average sum-rate}

For a more tractable sum-rate analysis, the expression in (9) can be approximated at high SNR as

$$
\begin{aligned}
\mathcal{R}_{k}^{\text {sum }} & \approx \frac{1}{2} \log \left(\gamma_{a, k} \gamma_{b, k}\right) \\
& \approx \log \left(\omega_{k}\right)+\log \left(\frac{P_{r} \sqrt{\Lambda_{k}}}{N_{0}}\right),
\end{aligned}
$$

where $\omega_{k}=\frac{\left|h_{a, k}\right|^{2}\left|h_{b, k}\right|^{2}}{\left|h_{a, k}\right|^{2}+\left|h_{b, k}\right|^{2}}$ is half of the harmonic mean of channel strengths $\left|h_{a, k}\right|^{2}$ and $\left|h_{b, k}\right|^{2}$, and $\Lambda_{k}=\frac{\left(\left|h_{a, k}\right|^{2}+\left|h_{b, k}\right|^{2}\right)^{2}}{\left(\eta\left|h_{a, k}\right|^{2}+\left|h_{b, k}\right|^{2}\right)\left(\eta\left|h_{b, k}\right|^{2}+\left|h_{a, k}\right|^{2}\right)}$.

By applying the bounds $\frac{2}{\eta^{2}+1}<\Lambda_{k}<\frac{1}{\eta}$ for all $k[9$, Theorem 2], and the well-known bounds for the harmonic mean as $\frac{1}{2} \min (x, y) \leq \frac{x y}{x+y}<\min (x, y)[24]$, we can bound the sum-rate for best pair bidirectional transmission at high SNR as

$$
\log \left(\phi_{k^{*}}\right)+\log \left(\frac{P_{r}}{N_{0} \sqrt{2\left(\eta^{2}+1\right)}}\right)<\mathcal{R}_{k^{*}}^{\text {sum }}<\log \left(\phi_{k^{*}}\right)+\log \left(\frac{P_{r}}{N_{0} \sqrt{\eta}}\right) .
$$

Therefore, the average sum-rate in (30) is bounded by

$$
\mathbb{E}\left[\log \left(\phi_{k^{2}}\right)\right]+\log \left(\frac{P_{r}}{N_{0} \sqrt{2\left(\eta^{2}+1\right)}}\right)<\overline{\mathcal{R}}_{k^{*}}^{\text {sum }}<\mathbb{E}\left[\log \left(\phi_{k^{\prime}}\right)\right]+\log \left(\frac{P_{r}}{N_{0} \sqrt{\eta}}\right)
$$

The expectation term in (31) can be evaluated as follows:

$$
\mathbb{E}\left[\log \left(\phi_{k^{*}}\right)\right]=\int_{0}^{\infty} \log (\phi) f_{\phi_{k^{*}}}(\phi) \mathrm{d} \phi,
$$

where $f_{\phi_{k^{*}}}(\phi)$ is the PDF of $\varphi_{k^{*}}$, which can be evaluated using (17) in (18) with $\theta$ replaced by $\varphi$ as

$$
f_{\phi_{k^{*}}}(\phi)=\sum_{k=1}^{K} \frac{1}{\bar{\phi}_{k}} \sum_{n=0}^{K-1}\left(\begin{array}{c}
K-1 \\
n
\end{array}\right)(-1)^{n} \mathrm{e}^{-(n+1) \phi / \bar{\phi}_{k}} .
$$

Substituting (33) into (32), we get

$$
\mathbb{E}\left[\log \left(\phi_{k^{*}}\right)\right]=\sum_{k=1}^{K} \frac{1}{\bar{\phi}_{k}} \sum_{n=0}^{K-1}\left(\begin{array}{c}
K-1 \\
n
\end{array}\right)(-1)^{n} \int_{0}^{\infty} \log (\phi) \mathrm{e}^{-(n+1) \phi / \bar{\phi}_{k}} \mathrm{~d} \phi .
$$

We can evaluate the integral term in (34) using [25, eq. 4.331.1] to obtain

$$
\mathbb{E}\left[\log \left(\phi_{k^{*}}\right)\right]=\log (e) \sum_{k=1}^{K} \sum_{n=0}^{K-1}\left(\begin{array}{c}
K-1 \\
n
\end{array}\right) \frac{(-1)^{n+1}}{n+1}\left[\mathrm{C}+\ln \left(\frac{n+1}{\bar{\phi}_{k}}\right)\right],
$$

where $\mathbf{C}=0.577215664 \ldots$ is Euler's constant defined by [25, eq. 8.367.1].

Knowing that $\sum_{n=0}^{K-1}\left(\begin{array}{c}K \\ n+1\end{array}\right)(-1)^{n+1}=-1$, we can express (35) as 


$$
\mathbb{E}\left[\log \left(\phi_{k^{*}}\right)\right]=\frac{1}{K} \sum_{k=1}^{K} \log \left(\bar{\phi}_{k}\right)+\zeta(K)-\mathrm{C} \log (e),
$$

where $\zeta(K)$ is a constant not related with SNR or link quality given by

$$
\zeta(K)=\sum_{n=0}^{K-1}\left(\begin{array}{c}
K \\
n+1
\end{array}\right)(-1)^{n+1} \log (n+1) .
$$

Inserting (36) into (31) and after invoking power assignment as considered previously, we obtain

$$
\begin{aligned}
\log \left[\frac{\varrho \alpha(1-\alpha)}{2(1+\alpha)}\right. & \left.+\frac{1}{K} \sum_{k=1}^{K} \log \left(\bar{\phi}_{k}\right)+\zeta(K)-\log \left(e^{\mathrm{C}}\right)<\overline{\mathcal{R}}_{k^{k}}^{\mathrm{sum}}\right] \\
& <\log \left[\varrho \alpha \sqrt{\frac{1-\alpha}{1+\alpha}}\right]+\frac{1}{K} \sum_{k=1}^{K} \log (\bar{\phi} k)+\zeta(K)-\log \left(e^{\mathrm{C}}\right) .
\end{aligned}
$$

As $\zeta(K)>\log \left(\mathrm{e}^{\mathrm{C}}\right)$ for $K \geq 2$, the average sum-rate increases with $K$. It can now be shown easily that the lower bound of average sum-rate is maximized when $\alpha$ $\approx 0.414$ (as for outage minimization), whereas the upper bound is maximized when $\alpha \approx 0.618$ (as in [9]).

\section{Numerical and simulation results}

In this section, we present numerical and simulation results to demonstrate the performance of the considered scheme. For numerical evaluations, we use selected channel variances as listed in Table 1 to reflect randomness in $K$ user pairs with nonidentical distributions. This is owing to the fact that different users may be placed at different distances from the relay, and hence, they may have different average SNR values. In the following numerical studies, we assume $\gamma_{\text {th }}=1$ (for outage probability).

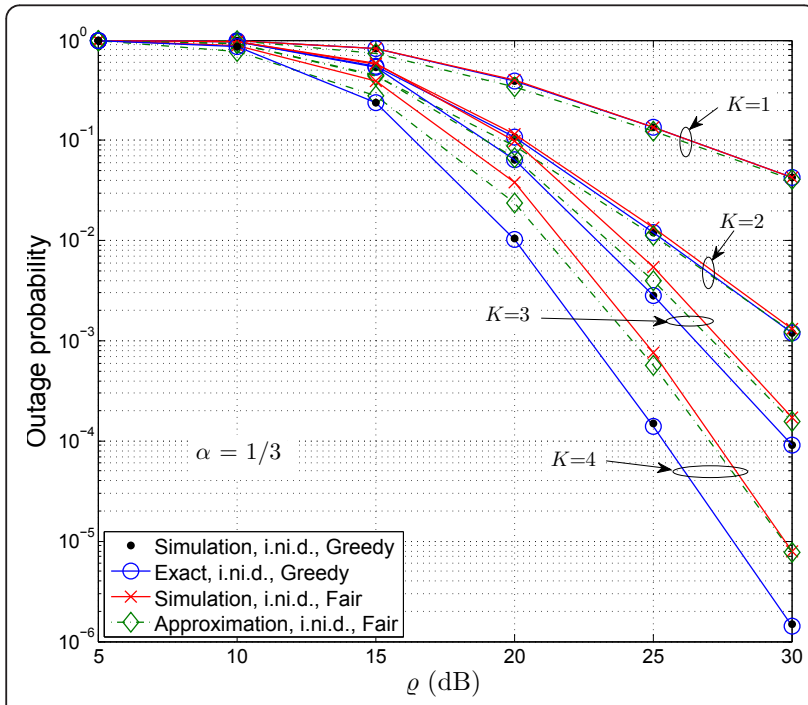

Figure 2 Outage probabilities of opportunistic scheduling scheme in multiuser two-way relaying with $K$ i.ni.d. pairs.
Figure 2 shows the outage probability curves for various user pairs with nonidentical $\bar{\theta}_{k}$ versus total SNR (Q) under uniform power distribution among the selected users and the relay $(\alpha=1 / 3)$. The exact curves corresponding to the evaluation of (25) for greedy scheduler were obtained by truncating the infinite series over index $n$ in (14) and (15) to first few terms beyond which there is no change in the first seven decimal places of the results. As can be seen from Figure 2, the exact curves match perfectly with the results generated through Monte Carlo simulations, validating our analytical expression. Further, it can be seen that the analytical outage curves for fair scheduling scheme corresponding to the computation of (26) closely approximate the simulated values when the SNR is large. This implies that our approximated expression in (26) can provide good predictions of outage probabilities for fair scheduling scheme in the high SNR regime. The curves in Figure 2, however, illustrate that the greedy scheduling scheme outperforms the fair one. This is expected since fairness in the scheduling scheme results in some performance loss. Moreover, it is obvious from this figure that the overall system attains a multiuser diversity of order $K$.

Figures 3 and 4 demonstrate the outage performance of greedy and fair scheduling schemes, respectively, with different power assignments to the selected relay (by varying $\alpha$ ) subject to a total power constraint. We can see that the minimum of the outage probability under both the schemes lies in the range of $\alpha$ between 0.4 and 0.5 , regardless of the values of $\varrho$ and channel parameters. Also, as the outage probability is not very sensitive to $\alpha$ in this range, $\alpha \approx 0.4$ provides the near-

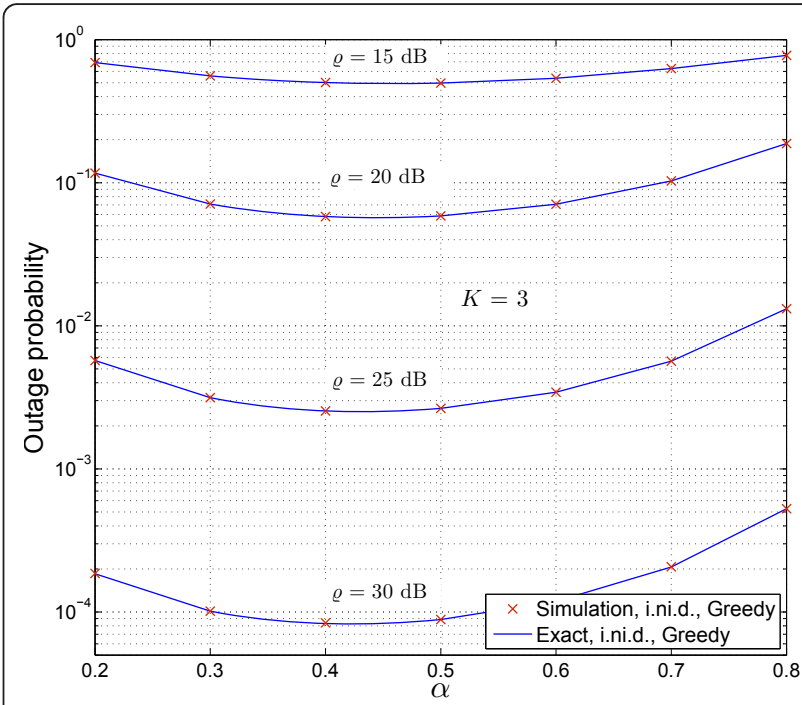

Figure 3 Outage probabilities of greedy scheduling scheme as a function of fraction of total power allocated to the relay. 


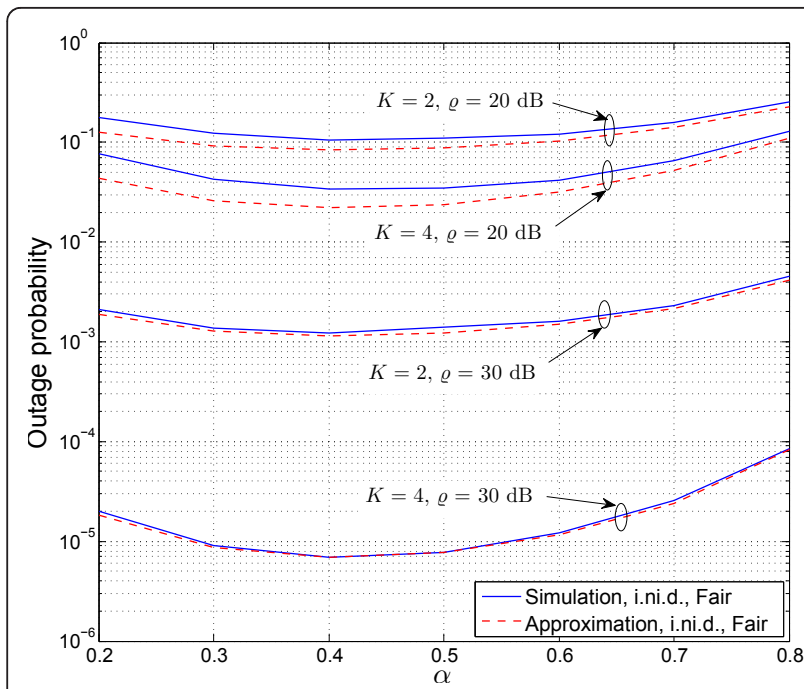

Figure 4 Outage probabilities of fair scheduling scheme as a function of fraction of total power allocated to the relay.

optimal performance for both the schemes. This is in good agreement with the result as derived analytically using the high SNR approximation in Section 4. It is an important result from a practical point of view since such power allocation scheme does not depend on the system/channel parameters.

Figure 5 provides a comparison between user pairs with nonidentical and identical distributions in terms of outage probability as a function of $K$. For a fair comparison, we set up the simulation for i.i.d. case by considering $\bar{\theta}=\frac{1}{K} \sum_{k=1}^{K} \bar{\theta}_{k}$ for all $k$ (as all the user pairs are required

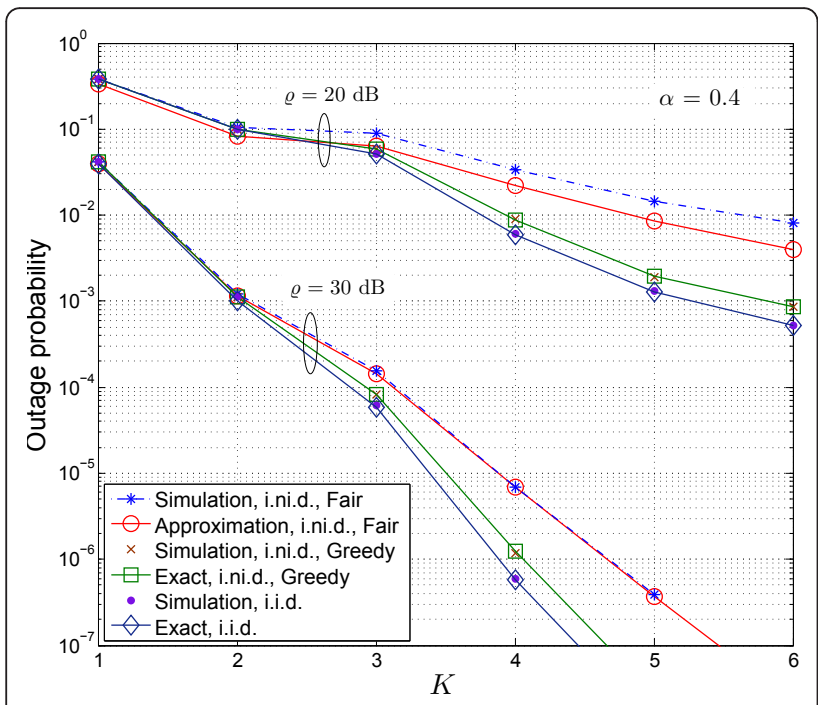

Figure 5 Comparison of outage probabilities for i.ni.d. and i.i.d. user pairs of opportunistic scheduling scheme in multiuser two-way relaying. to have same statistics), so that the sum of $\bar{\theta}_{k}$ s is equal to $K \bar{\theta}$ under both cases. Note that for i.i.d. user pairs, both greedy and fair scheduling schemes have the same performance, as stated earlier. It can be observed that i.i.d. pairs achieve better performance than i.ni.d. ones.

With the same set of parameters, we plot the average sum-rate curves versus the number of pairs $K$ in Figure 6. This figure shows that the average sum-rate performance for two-way relaying can also be improved by including more user pairs in the considered scheme. There is a gap between the bounds and simulation curves throughout the region of high SNR. This is because at high SNR, both $\gamma_{a, k}$ and $\gamma_{b, k}$ have a high probability of having values close to each other, and hence, their harmonic mean does not approximate its upper or lower bound very well. Figure 6 also illustrates that the average sum-rate performance of $i$. i.d. user pairs is better than that of i.ni.d. pairs.

Figures 7 and 8 present a comparison of the performance of the proposed scheme with that of the direct transmission scheme using same scheduling procedure in terms of outage probability and average sum-rate, respectively, as a function of the distance $d_{a b}$ between two users of the best selected pair. We set up the simulation by considering an i.i.d. case with relay location lies midway between the two users so that $d_{a b}=2 d_{a}=2 d_{b}$, where $d_{a}$ and $d_{b}$ represent the distances of $T_{a, k}$ and $T_{b, k}$ from $R$, respectively, for all $k$. We incorporate the large-scale path loss in the signal propagation with a path loss exponent $v$. As such, we can have $\sigma_{a, k}^{2}=d_{a}^{-v}, \sigma_{b, k}^{2}=d_{b}^{-v}$, and $\sigma_{a b, k}^{2}=d_{a b}^{-v}$ for all $k$, where $\sigma_{a b, k}^{2}$ denotes the channel variance of direct link between $T_{a, k}$ and $T_{b, k}$. We assume equal power $P$ at all nodes in the network. Further, we consider radio

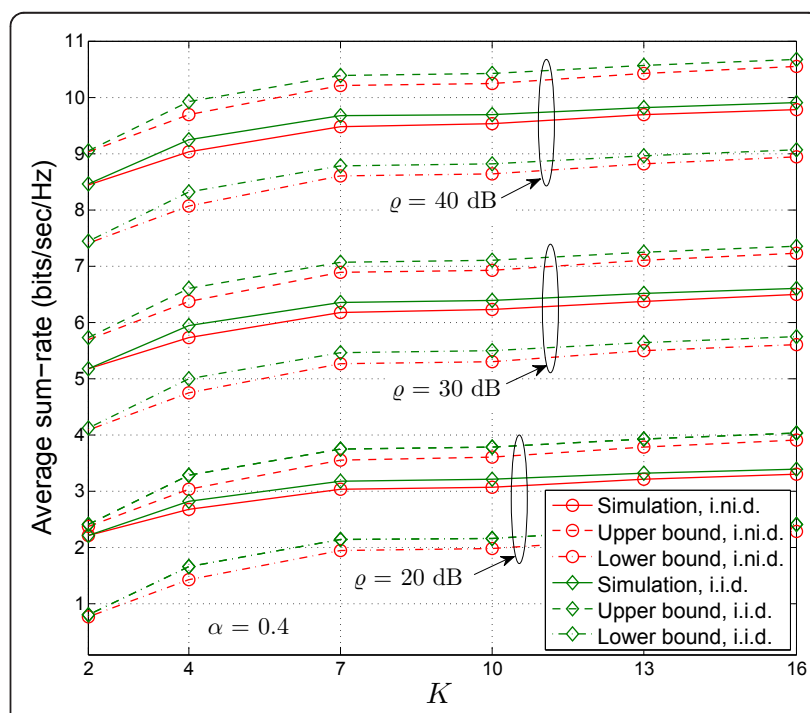

Figure 6 Comparison of average sum-rates for i.ni.d. and i.i.d. user pairs of opportunistic scheduling scheme with fairness in multiuser two-way relaying. 


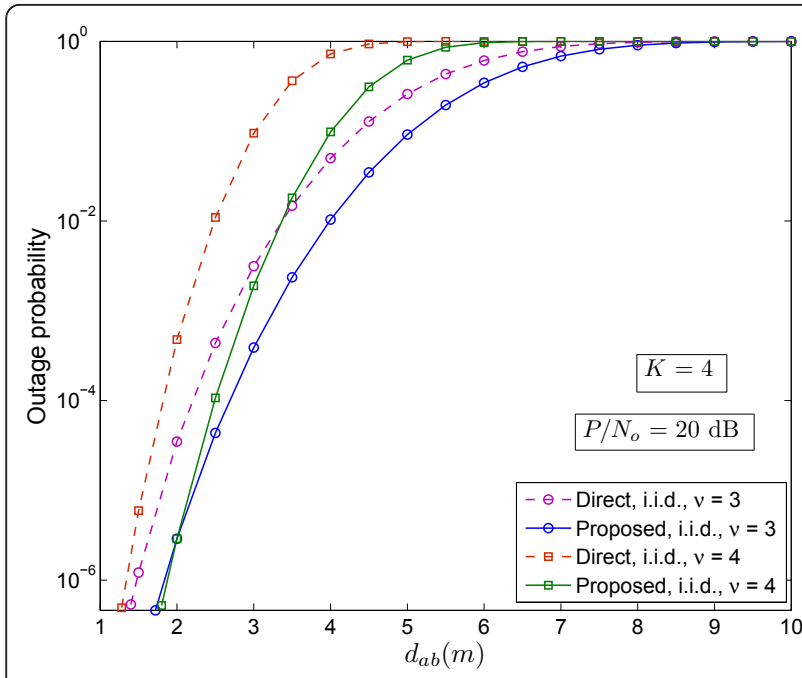

Figure 7 Comparison of outage performance of proposed scheduling scheme with direct transmission for i.i.d. user pairs.

propagation with $v=3,4$ in practical cases of highly shadowed environment [26]. It can be seen from these figures that the performance of both schemes will degrade with the increasing distance between the users of the selected pair, as expected. However, it is interesting to observe that the considered two-way relaying-based scheduling scheme performs much better than the direct transmission-based scheme in a practical shadowed environment.

\section{Conclusion and future work}

We have investigated the performance of an outage-optimal opportunistic scheduling scheme with fairness for a multi-pair ANC-based two-way relay network over a

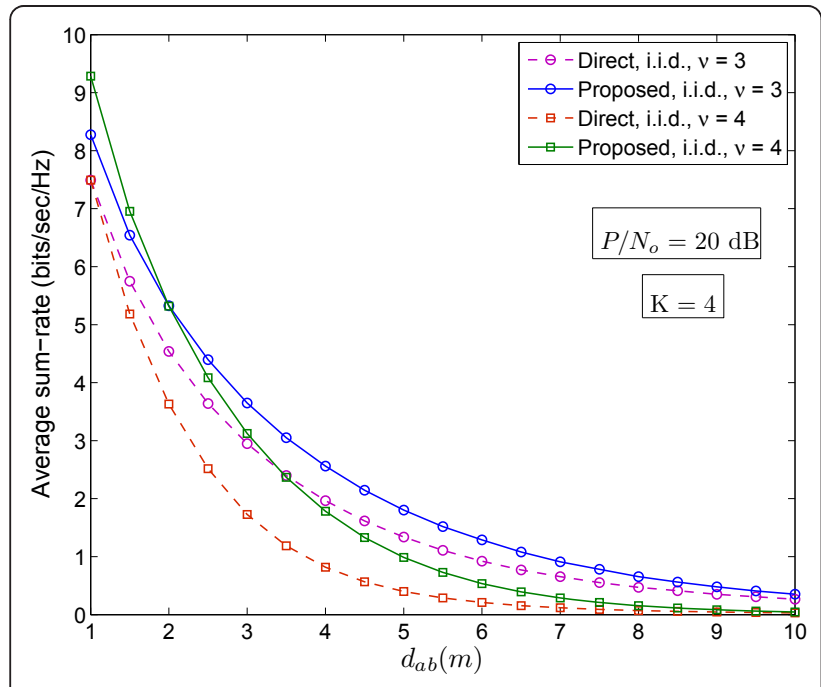

Figure 8 Comparison of average sum-rate performance of proposed scheduling scheme with direct transmission for i.i.d. user pairs
Rayleigh flat-fading scenario. For the greedy scheduling scheme of user pairs, we derived an exact expression for the outage probability that is valid over entire SNR region. We then proposed a scheduling strategy that ensures fairness among user pairs of the considered system. Based on a high SNR assumption, we derived an approximate expression for the outage probability and the bounds on the average sum-rate of the overall system. It was shown that the proposed scheme achieves performance gain by attaining an order of multiuser diversity equal to the number of user pairs. It is further demonstrated that near-optimal performance can be achieved when about $40 \%$ of the available power is assigned to the relay, irrespective of the system parameters.

In the present work, we have analyzed the considered scheduling scheme by assuming perfect channel estimation and no delay between the instants of estimation and best pair transmission. However, estimation errors and scheduling delays do exist in practical systems, and analyzing their effects on the performance is a subject for future work.

\section{Appendix I}

\section{Proof of Theorem 1}

We can express

$$
\operatorname{Pr}\left[\min \left(\gamma_{a, k}, \gamma_{b, k}\right)<\theta\right]=P_{1, k}+P_{2, k},
$$

where $P_{1, k}=\operatorname{Pr}\left[\gamma_{b, k}<\theta, \gamma_{b, k}<\gamma_{a, k}\right]$ and $P_{2, k}=\operatorname{Pr}\left[\gamma_{a, k}<\theta\right.$, $\left.\gamma_{a, k}<\gamma_{b, k}\right]$. Using (7), and (8) and after some straightforward manipulations, we can re-express

$$
P_{1, k}=\operatorname{Pr}\left[\frac{x_{1, k} y_{1, k}}{x_{1, k}+y_{1, k}+1}<\gamma, x_{1, k}<\frac{y_{1, k}}{\eta}\right],
$$

where $x_{1, k} \triangleq \frac{P}{N_{0}}\left|h_{a, k}\right|^{2}$ and $y_{1, k} \triangleq \frac{\left(P+P_{r}\right)}{N_{0}}\left|h_{b, k}\right|^{2}$. For Rayleigh fading, $x_{1, k}$ and $y_{1, k}$ are exponentially distributed random variables with parameters $\lambda_{x_{1, k}}=N_{0} /\left(P \sigma_{a, k}^{2}\right)$ and $\lambda_{y_{1, k}}=N_{0} /\left(\left(P+P_{r}\right) \sigma_{b, k}^{2}\right)$, and probability density functions $\quad f_{x_{1, k}}(x)=\lambda_{x_{1, k}} \mathrm{e}^{-\lambda x_{1, k} x}, x \geq 0 \quad$ and $f_{\gamma_{1, k}}(y)=\lambda_{y_{1, k}} \mathrm{e}^{-\lambda y_{1, k} \gamma}, y \geq 0$, respectively. Now (40) can, be evaluated as follows:

$$
\begin{aligned}
P_{1, k} & =\operatorname{Pr}\left[x_{1, k}\left(y_{1, k}-\gamma\right)<\gamma\left(\gamma_{1, k}+1\right), \eta x_{1, k}<\gamma_{1, k}\right] \\
& =\int_{0}^{\gamma} f_{\gamma_{1, k}}(\gamma) \int_{0}^{\frac{\gamma}{\eta}} f_{x_{1, k}}(x) \mathrm{d} x \mathrm{~d} y+\int_{\gamma}^{\infty} f_{\gamma_{1, k}}(\gamma) \int_{0}^{\min \left(\frac{\gamma}{\eta}, \frac{\gamma(\gamma+1)}{\gamma-\gamma}\right)} f_{x_{1, k}}(x) \mathrm{d} x \mathrm{~d} \gamma .
\end{aligned}
$$

It can be shown that $\frac{\gamma}{\eta}<\frac{\gamma(\gamma+1)}{\gamma-\gamma}$ for $y$ lying in the range $\gamma<y<\delta$, where $\delta$ can be obtained by solving $y^{2}-\gamma$ $(\eta+1) y-\gamma \eta 0$ for $y$ with $\eta>1$ as the possible root. Hence the second term in (41) can be separated into two parts to yield 


$$
\begin{aligned}
P_{1, k} & =\int_{0}^{\gamma} f_{y_{1, k}}(y) \int_{0}^{\frac{\gamma}{\eta}} f_{x_{1, k}}(x) \mathrm{d} x \mathrm{~d} y+\int_{\gamma}^{\delta} f_{y_{1, k}}(y) \int_{0}^{\frac{\gamma}{\eta}} f_{x_{1, k}}(x) \mathrm{d} x \mathrm{~d} y \\
& +\int_{\delta}^{\infty} f_{y_{1, k}}(y) \int_{0}^{\frac{\gamma(\gamma+1)}{\gamma-\gamma}} f_{x_{1, k}}(x) \mathrm{d} x \mathrm{~d} y_{,}
\end{aligned}
$$

which can be simplified further as

$$
P_{1, k}=\int_{0}^{\delta} f_{y_{1, k}}(y) \int_{0}^{\frac{y}{\eta}} f_{x_{1, k}}(x) \mathrm{d} x \mathrm{~d} y+\int_{\delta}^{\infty} f_{y_{1, k}}(y) \int_{0}^{\frac{\gamma(y+1)}{\gamma-\gamma}} f_{x_{1, k}}(x) \mathrm{d} x \mathrm{~d} y
$$

where

$$
\begin{aligned}
& I_{1} \triangleq \int_{0}^{\delta} \lambda_{y_{1, k}} \mathrm{e}^{-\lambda_{y_{1, k} y}} \int_{0}^{\frac{y}{\eta}} \lambda_{x_{1, k}} \mathrm{e}^{-\lambda_{x_{1, k}} x} \mathrm{~d} x \mathrm{~d} y \\
& =\int_{0}^{\delta} \lambda_{y_{1, k}} \mathrm{e}^{-\lambda_{y_{1, k}} y}\left(1-\mathrm{e}^{-\frac{\lambda_{x_{1, k}}}{\eta} y}\right) \mathrm{d} y \\
& =1-\mathrm{e}^{-\lambda_{y_{1, k} \delta}}-\frac{\eta \lambda_{y_{1, k}}\left[1-\mathrm{e}^{-\frac{\delta}{\eta}\left(\lambda_{x_{1, k}}+\eta \lambda_{\gamma_{1, k}}\right)}\right]}{\lambda_{x_{1, k}}+\eta \lambda_{y_{1, k}}}
\end{aligned}
$$

and

$$
\begin{aligned}
I_{2} & \triangleq \int_{\delta}^{\infty} \lambda_{y_{1, k}} \mathrm{e}^{-\lambda_{y_{1, k} \gamma}} \int_{0}^{\frac{\gamma(\gamma+1)}{y-\gamma} \lambda_{x_{1, k}} \mathrm{e}^{-\lambda_{x_{1, k} x}} \mathrm{~d} x \mathrm{~d} y} \\
& =\int_{\delta}^{\infty} \lambda_{y_{1, k}} \mathrm{e}^{-\lambda_{y_{1, k} \gamma}}\left(1-\mathrm{e}^{-\lambda_{x_{1, k}} \gamma\left(\frac{y+1}{y-1}\right)}\right) \mathrm{d} y \\
& =\mathrm{e}^{-\lambda_{y_{1, k} \delta}}-\int_{\delta}^{\infty} \lambda_{y_{1, k}} \mathrm{e}^{-\lambda_{y_{1, k} \gamma}} \mathrm{e}^{-\lambda_{x_{1, k}} \gamma\left(\frac{y+1}{y-\gamma}\right)} \mathrm{d} \gamma .
\end{aligned}
$$

Operating the change of variable $t=y-\gamma$ within the integral in (45) and some simplifications, we get

$$
I_{2}=\mathrm{e}^{-\lambda_{y_{1, k}}}-\lambda_{y_{1, k}} \mathrm{e}^{-\gamma\left(\lambda_{x_{1, k}}+\lambda_{\gamma_{1, k}}\right)} \int_{\delta-\gamma}^{\infty} \mathrm{e}^{-\lambda_{y_{1, k}} t} \mathrm{e}^{-\lambda_{x_{1, k}} \gamma\left(\frac{\gamma+1}{t}\right)} \mathrm{d} t(46)
$$

Since the integral in (46) has no closed-form solution, it can be evaluated by using Taylor series expansion [25, eq. 1.211.1] for the second exponential term and interchanging the order of integration and summation as

$$
\begin{aligned}
I_{2} & =\mathrm{e}^{-\lambda_{\gamma_{1, k}} \delta^{\delta}}-\lambda_{y_{1, k}} \mathrm{e}^{-\gamma\left(\lambda_{x_{1, k}}+\lambda_{\gamma_{1, k}}\right)} \sum_{n=0}^{\infty} \frac{\left[-\lambda_{x_{1, k}, k} \gamma(\gamma+1)\right]^{n}}{n !} \int_{\delta-\gamma}^{\infty} \frac{\mathrm{e}^{-\lambda_{\gamma_{1, k}} t}}{t^{n}} \mathrm{~d} t \\
& =\mathrm{e}^{-\lambda_{y_{1, k}}}-\lambda_{\gamma_{1, k}}(\delta-\gamma) \mathrm{e}^{-\gamma\left(\lambda_{x_{1, k}}+\lambda_{\gamma_{1, k}}\right)} \sum_{n=0}^{\infty} \frac{(-1)^{n}}{n !}\left[\frac{\lambda_{x_{1, k}} \gamma(\gamma+1)}{\delta-\gamma}\right]^{n} \\
& \times E_{n}\left[\lambda_{\gamma_{1, k}}(\delta-\gamma)\right],
\end{aligned}
$$

where the last equality follows from [21, eq. 5.1.4] after a simple transformation of the integration variable. Substituting (44) and (47) into (43) yields the expression of $P_{1, k}$ as provided in (14). Following the similar steps as above, we obtain $P_{2, k}$ as presented in (15). And the proof of Theorem 1 is completed.

\section{Appendix II}

\section{Proof of Lemma 1}

The end-to-end instantaneous SNR expressions in (7) and (8) can be approximated at high SNR $\left(P \gg N_{0}\right)$, respectively, by

$$
\gamma_{a, k} \approx \frac{P_{r}}{N_{0}}\left[\frac{\left|h_{a, k}\right|^{2}\left|h_{b, k}\right|^{2}}{\eta\left|h_{a, k}\right|^{2}+\left|h_{b, k}\right|^{2}}\right]
$$

and

$$
\gamma_{b, k} \approx \frac{P_{r}}{N_{0}}\left[\frac{\left|h_{a, k}\right|^{2}\left|h_{b, k}\right|^{2}}{\eta\left|h_{b, k}\right|^{2}+\left|h_{a, k}\right|^{2}}\right],
$$

where the approximation is made by ignoring the noise power in the gain at the relay. Despite this, such an approximation has been shown to be very tight in the entire SNR region $[9,13,24]$. Using these SNR expressions and following the similar approach as in "Appendix I," we can express $P_{1, k}$ in (14) as

$$
\begin{aligned}
P_{1, k} \approx & 1-\frac{\sigma_{a, k}^{2}}{\sigma_{a, k}^{2}+\sigma_{b, k}^{2}}\left[1-e^{\left.-\frac{\theta \eta N_{0}}{P_{r}}\left(1+\frac{1}{\eta}\right)\left(\frac{1}{\sigma_{a, k}^{2}}+\frac{1}{\sigma_{b, k}^{2}}\right)\right]}\right. \\
& -\frac{\theta \eta N_{0}}{P_{r} \sigma_{b, k}^{2}} e^{-\frac{\theta \eta N_{0}}{P_{r}}\left(\frac{1}{\sigma_{a, k}^{2}}+\frac{1}{\eta \sigma_{b, k}^{2}}\right)} \sum_{n=0}^{\infty} \frac{(-1)^{n}}{n !}\left(\frac{\theta N_{0}}{P_{r} \sigma_{b, k}^{2}}\right)^{n} E_{n}\left[\frac{\theta \eta N_{0}}{P_{r} \sigma_{b, k}^{2}}\right] .
\end{aligned}
$$

Now for $n \geq 1, E_{n}[z]$ can be expanded in series as [21, eq. 5.1.12]

$$
E_{n}[z]=\frac{(-z)^{n-1}}{(n-1) !}(\psi(n)-\ln (z))-\sum_{\substack{q=0 \\ q \neq n-1}}^{\infty} \frac{(-z)^{q}}{(q-n+1) q !}
$$

where $\psi(n)=-\mathbf{C}+\sum_{s=1}^{n-1} \frac{1}{s}, n>1$ and $\psi(1)=-\mathbf{C}$, where $\mathbf{C}=0.577215664 \ldots$ is Euler's constant. By using (51), one can verify that for high $\operatorname{SNR}\left(P / N_{0} \rightarrow \infty\right)$ and the fact that $\lim _{z \rightarrow \infty} \frac{\ln (1 / z)}{z^{2}}=0$, the summation term for $n$ 
$\geq 1$ in (50) becomes infinitesimal of order $2 n$. Therefore, we can write

$$
\begin{aligned}
P_{1, k} & \approx 1-\frac{\sigma_{a, k}^{2}}{\sigma_{a, k}^{2}+\sigma_{b, k}^{2}}\left[1-\mathrm{e}^{-\frac{\theta \eta N_{0}}{P_{r}}\left(1+\frac{1}{\eta}\right)\left(\frac{1}{\sigma_{a, k}^{2}}+\frac{1}{\sigma_{b, k}^{2}}\right)}\right] \\
& -\frac{\theta \eta N_{0}}{P_{r} \sigma_{b, k}^{2}} e^{-\frac{\theta \eta N_{0}}{P_{r}}\left(\frac{1}{\sigma_{a, k}^{2}}+\frac{1}{\eta \sigma_{b, k}^{2}}\right)} E_{0}\left[\frac{\theta \eta N_{0}}{P_{r} \sigma_{b, k}^{2}}\right]
\end{aligned}
$$

where we have omitted the higher-order terms. Further, with $E_{0}[z]=e^{-z} / z$, we can express (52) for large SNR as

$$
\begin{aligned}
P_{1, k} & \approx 1-\frac{\sigma_{a, k}^{2}}{\sigma_{a, k}^{2}+\sigma_{b, k}^{2}}\left[1-\mathrm{e}^{-\frac{\theta \eta N_{0}}{P_{r}}\left(1+\frac{1}{\eta}\right)\left(\frac{1}{\sigma_{a, k}^{2}}+\frac{1}{\sigma_{b, k}^{2}}\right)}\right] \\
& -\mathrm{e}^{-\frac{\theta \eta N_{0}}{P_{r}}\left(\frac{1}{\sigma_{a, k}^{2}}+\frac{1}{\eta \sigma_{b, k}^{2}}\right)} \mathrm{e}^{-\frac{\theta \eta N_{0}}{P_{r} \sigma_{b, k}^{2}}}
\end{aligned}
$$

Similarly, we approximate $P_{2, k}$ in (15) for large SNR as

$$
\begin{aligned}
P_{2, k} \approx & 1-\frac{\sigma_{b, k}^{2}}{\sigma_{a, k}^{2}+\sigma_{b, k}^{2}}\left[1-\mathrm{e}^{-\frac{\theta \eta N_{0}}{P_{r}}\left(1+\frac{1}{\eta}\right)\left(\frac{1}{\sigma_{a, k}^{2}}+\frac{1}{\sigma_{b, k}^{2}}\right)}\right] \\
& -\mathrm{e}^{-\frac{\theta \eta N_{0}}{P_{r}}\left(\frac{1}{\sigma_{b, k}^{2}}+\frac{1}{\eta \sigma_{a, k}^{2}}\right)} \mathrm{e}^{-\frac{\theta \eta N_{0}}{P_{r} \sigma_{a, k}^{2}}}
\end{aligned}
$$

Substituting (53) and (54) into (13), the approximate expression for $F_{\theta_{k}}(\theta)$ at the high $\mathrm{SNR}$ region is given by

$$
F_{\theta_{k}}(\theta) \approx 1-\mathrm{e}^{-\frac{\theta \eta N_{0}}{P_{r}}\left(\frac{1}{\sigma_{a, k}^{2}}+\frac{1}{\sigma_{b, k}^{2}}\right)}\left[\mathrm{e}^{-\frac{\theta N_{0}}{P_{r} \sigma_{a, k}^{2}}}+\mathrm{e}^{-\frac{\theta N_{0}}{P_{r} \sigma_{b, k}^{2}}}-\mathrm{e}^{-\frac{\theta N_{0}}{P_{r}}\left(\frac{1}{\sigma_{a, k}^{2}}+\frac{1}{\sigma_{b, k}^{2}}\right)}\right] .
$$

Note that the above expression is dominated by the exponential term present outside the square brackets due to the factor $\eta>1$. Therefore, by making use of the fact that $\mathrm{e}^{-z} \underset{z \rightarrow 0}{\approx} 1-z$ for the terms within square brackets, we can express the result as given in (16).

\section{Additional material}

Additional file 1: 1597702927618362_LE[1].pdf, 310K http://jwCn.

eurasipjournals.com/imedia/1896637463640433/supp1.pdf

\section{Acknowledgements}

This work was supported in part by the Department of Science and Technology, Government of India, under Project SR/S3/EECE/031/2008. A conference version with initial results pertaining to this paper has been presented at IEEE Wireless Communications and Networking Conference (WCNC), Can-cun, Mexico, March 2011. The authors would like to thank the anonymous reviewers for their helpful suggestions and insightful comments.

\section{Author details}

'Department of Electrical Engineering, Indian Institute of Technology Delhi, New Delhi, 110016, India ²Department of Electrical Engineering, Indian Institute of Technology Delhi, New Delhi, 110016, India

\section{Competing interests}

The authors declare that they have no competing interests.

Received: 22 December 2010 Accepted: 5 December 2011 Published: 5 December 2011

\section{References}

1. JN Laneman, DNC Tse, GW Wornell, Cooperative diversity in wireless networks: efficient protocols and outage behavior. IEEE Trans Inform Theory 50(12), 3062-3080 (2004). doi:10.1109/TIT.2004.838089

2. R Ahlswede, N Cai, SYR Li, RW Yeung, Network information flow. IEEE Trans Inform Theory 46(4), 1204-1216 (2000). doi:10.1109/18.850663

3. Y Wu, P Chou, SY Kung, Information exchange in wireless networks with network coding and physical-layer broadcast, in Proceedings of the 39th Annual Conference on Information Sciences and Systems (CISS). Baltimore, Maryland (2005)

4. P Larsson, N Johansson, KE Sunell, Coded bidirectional relaying, in Proceedings of the IEEE VTC, Melbourne (2006)

5. P Popovski, $\mathrm{H}$ Yomo, Bi-directional amplification of throughput in a wireless multihop network, in Proceedings of the IEEE VTC, Melbourne (2006)

6. S Zhang, SC Liew, PP Lam, Physical-layer network coding, in Proceedings of the ACM MobiCom, Los Angeles, 358-365 (2006)

7. SJ Kim, P Mitran, $V$ Tarokh, Performance bounds for bi-directional coded cooperation protocols. IEEE Trans Inform Theory. 54(11), 5235-5241 (2008)

8. B Rankov, A Wittneben, Spectral efficient protocols for half-duplex relay channels. IEEE J Select Areas Commun. 25(2), 379-389 (2007)

9. Y Han, SH Ting, CK Ho, WH Chin, Performance bounds for two-way amplifyand-forward relaying. IEEE Trans Wireless Commun. 8(1), 432-439 (2009)

10. S Katti, S Gollakota, D Katabi, Embracing wireless interference: analog network coding. in Proceedings of the ACM SIGCOMM, 397-408 (2007)

11. R Zhang, YC Liang, CC Chai, S Cui, Optimal beamforming for two-way multi-antenna relay channel with analogue network coding. IEEE J Select Areas Commun. 27(5), 699-712 (2009)

12. P Popovski, H Yomo, Wireless network coding by amplify-and-forward for bi-directional traffic flows. IEEE Commun Lett. 11(1), 16-18 (2007)

13. Z Yi, I-M Kim, R Schober, Finite-SNR diversity-multiplexing tradeoff in bidirectional cooperative networks, in Proceedings of the IEEE ICC, Cape Town (2010)

14. C Esli, A Wittneben, One- and two-way decode-and-forward relaying for wireless multiuser MIMO networks, in Proceedings of IEEE Globecom, New Orleans (2008)

15. M Chen, A Yener, Multiuser two-way relaying: detection and interference management strategies. IEEE Trans Wireless Commun. 8(8), 4296-4305 (2009)

16. S Avestimehr, A Khajehnejad, A Sezgin, B Hassibi, Capacity region of the deterministic multi-pair bi-directional relay network, in Proceedings of IEEE ITW, Greece (2009)

17. A Sezgin, A Khajehnejad, S Avestimehr, B Hassibi, Approximate capacity region of the two-pair bidirectional Gaussian relay network, in Proceedings of the IEEE ISIT 2009, Korea (2009)

18. E Yilmaz, R Zakhour, D Gesbert, R Knopp, Multi-pair two-way relay channel with multiple antenna relay station, in Proceedings of the IEEE ICC, Cape Town (2010)

19. P Viswanath, D Tse, R Laroia, Opportunistic beamforming using dumb antennas. IEEE Trans Inform Theory 48(6), 1277-1294 (2002). doi:10.1109/ TIT.2002.1003822

20. L Yang, MS Alouini, Performance analysis of multiuser selection diversity. IEEE Trans Veh Technol. 55(6), 1848-1861 (2006)

21. M Abramowitz, IA Stegun, Handbook of Mathematical Functions with Formulas, Graphs, and Mathematical Tables, 10th edn. (Dover Publications, New York, 1972)

22. W Rudin, Principles of Mathematical Analysis, 3rd edn. (McGraw-Hill, New York, 1976)

23. HA David, HN Nagaraja, Order Statistics, 3rd edn. (Wiley, New York, 2003) 
24. PA Anghel, M Kaveh, Exact symbol error probability of a cooperative network in a Rayleigh-fading environment. IEEE Trans Wireless Commun. 3(5), 1416-1421 (2004). doi:10.1109/TWC.2004.833431

25. IS Gradshteyn, IM Ryzhik, Tables of Integrals, Series and Products, 5th edn. (Academic Press, New York, 1994)

26. TS Rappaport, Wireless Communications: Principle and Practice (Prentice-Hall, Upper Saddle River, 2002)

doi:10.1186/1687-1499-2011-194

Cite this article as: Upadhyay and Prakriya: Outage-optimal opportunistic scheduling with analog network coding in multiuser two-way relay networks. EURASIP Journal on Wireless Communications and Networking 2011 2011:194.

\section{Submit your manuscript to a SpringerOpen ${ }^{\oplus}$} journal and benefit from:

- Convenient online submission

- Rigorous peer review

- Immediate publication on acceptance

- Open access: articles freely available online

- High visibility within the field

- Retaining the copyright to your article

Submit your next manuscript at $\gg$ springeropen.com 